FTUV-10-1117

KA-TP-25-2010

SFB/CPP-10-112

TTK-10-51

\title{
GLuOn-Fusion CONTRIBUtions to $\Phi+2$ Jet PRODUCTION
}

\author{
F. Campanario ${ }^{1}$, M. Kubocz ${ }^{2}$ and D. Zeppenfeld ${ }^{1}$ \\ ${ }^{1}$ Institut für Theoretische Physik, Universität Karlsruhe P.O.Box 698076128 \\ Karlsruhe, Germany \\ ${ }^{2}$ Institut für Theoretische Teilchenphysik und Kosmologie, RWTH Aachen University, \\ D52056 Aachen, Germany
}

\begin{abstract}
In high energy hadronic collisions a scalar or pseudoscalar Higgs boson, $\Phi=H$, $A$, can be efficiently produced via gluon fusion, which is mediated by heavy quark loops. We here consider double real emission corrections to $\Phi=A$ production, which lead to a Higgs plus two-jet final state, at order $\alpha_{s}^{4}$. Full quark mass effects are considered in the calculation of scattering amplitudes for the $\mathcal{C P}$-odd Higgs boson $A$, as induced by quark triangle-, box- and pentagon-diagrams. They complement the analogous results for a $\mathcal{C} \mathcal{P}$-even Higgs boson $H$ in Ref. 11. Interference effects between loops with top and bottom quarks as well as between $\mathcal{C P}$-even and $\mathcal{C P}$-odd couplings of the heavy quarks are fully taken into account.
\end{abstract}

October 30, $2018-1: 10$ 


\section{Introduction}

One of the prime tasks of the CERN Large Hadron Collider (LHC) is the search for the origins of the spontaneous breaking of the electroweak $S U(2) \times U(1)$ gauge symmetry and, once such particles are found, the study of one or several Higgs bosons as the remnants of the symmetry breaking mechanism. Among the various Higgs boson production channels the gluon fusion and the weak boson fusion processes have emerged as the most promising channels for Higgs boson discovery at the LHC [2, 3, 4], and they are equally valuable for the study of its properties, like the measurement of its couplings to gauge boson and fermions [5, 6].

In weak boson fusion (WBF), $q q \rightarrow q q H$ mediated by $t$-channel $W$ or $Z$ exchange, the two forward tagging jets arising from the scattered quarks provide a tell-tale signature which can be used for efficient background rejection [7]. The same $H j j$ signature can also arise in gluon fusion events, via $\mathcal{O}\left(\alpha_{s}^{2}\right)$ real emission corrections to $g g \rightarrow H$ which, within the Standard Model (SM), is mediated mainly by a top quark loop. For a Higgs boson which is lighter than the top quark, the resulting $H j j$ cross section can be determined to good approximation by an effective Lagrangian of energy dimension five, which is given by [8, 9]

$$
\mathcal{L}_{\text {eff }}=\frac{y_{t}}{y_{t}^{S M}} \cdot \frac{\alpha_{s}}{12 \pi v} \cdot H G_{\mu \nu}^{a} G^{a \mu \nu}+\frac{\tilde{y}_{t}}{y_{t}^{S M}} \cdot \frac{\alpha_{s}}{8 \pi v} \cdot A G_{\mu \nu}^{a} \tilde{G}^{a \mu \nu}
$$

where $G_{\mu \nu}^{a}$ denotes the gluon field strength and $\tilde{G}^{a \mu \nu}=1 / 2 G_{\rho \sigma}^{a} \varepsilon^{\mu \nu \rho \sigma}$ its dual. The two terms result from a $\bar{t} t H$ and a $\bar{t} \mathrm{i} \gamma_{5} t A$ coupling of the (pseudo)scalar Higgs, respectively, and they lead to distinctively different distributions of the azimuthal angle between the two jets: the $\mathcal{C P}$-even $\mathrm{Hg}$ g coupling produces a minimum for $\phi_{j j}= \pm \pi / 2$ while a $\mathcal{C P}$-odd Agg coupling leads to minima at $\phi_{j j}=0$ and $\pm \pi$. These distinctions become important in two-Higgs-doublet models (2HDM) like the minimal supersymmetric extension of the standard model (MSSM), where a $\mathcal{C} \mathcal{P}$-odd Higgs, $A$, appears in addition to a light and a heavy, neutral $\mathcal{C P}$-even Higgs, $h$ and $H$ : the azimuthal angle distribution of $\Phi j j$ events allow to differentiate between a $\mathcal{C P}$-even Higgs $(\Phi=h, H)$ or a $\mathcal{C P}$-odd one $(\Phi=A)$.

For large Higgs boson masses $\left(m_{H} \gtrsim m_{t}\right)$ the full quark mass dependence of the loop diagrams must be calculated for reliable predictions, and the same is true for large ratios of the two vacuum expectation values, $v_{u} / v_{d}=\tan \beta$, where bottom quark loops provide the dominant contributions to $q q \rightarrow q q H, q g \rightarrow q g H$ and $g g \rightarrow g g H$ amplitudes. For a $\mathcal{C P}$-even Higgs boson, these calculations were performed in Ref. [1]. The purpose of the present paper is to present the corresponding results for a $\mathcal{C} \mathcal{P}$-odd Higgs boson, $\Phi=A$, or, more precisely for an underlying Higgs coupling to quarks derived from the Yukawa Lagrangian $\mathcal{L}=y_{q} \bar{q} \mathrm{i} \gamma_{5} q A$. By combining the present results with those for a $\mathcal{C} \mathcal{P}$-even Higgs, the quark loop induced contributions to $\Phi j j$ production can be calculated for an arbitrary Yukawa coupling of the form

$$
\mathcal{L}_{\text {Yukawa }}=\bar{q}\left(y_{q}+\mathrm{i} \gamma_{5} \tilde{y}_{q}\right) q \Phi .
$$

Our results are implemented in a parton level Monte Carlo program which is part of the VBFNLO program package [10]. This numerical implementation allows to calculate 
$\Phi j j$ production cross sections in hadronic collisions including top- and bottom-quark loop contributions for arbitrary combinations of the Yukawa couplings $y_{q}$ and $\tilde{y}_{q}(q=t, b)$.

Our paper is organized as follows. In Section 2 we first define the models in which we consider pseudoscalar Higgs production. We then provide an outline of the calculation of the scattering amplitudes for the three basic subprocesses, $q q \rightarrow q q A, q g \rightarrow q g A$ and $g g \rightarrow g g A$. Further details on the various loop contributions are relegated to the Appendices. We have performed a number of analytic and numerical consistency checks on our calculation: they are described in Section 3. The main phenomenological results are presented in Section 4 , for $p p$ scattering at the LHC with a center of mass energy of $\sqrt{s}=14 \mathrm{TeV}$. For various combinations of top- and bottom quark contributions, parameterized by $\tan \beta$, we provide integrated $A j j$ cross sections but also differential distributions. Results are also presented for general $\Phi j j$ events, i.e. for the production of a Higgs boson with arbitrary $\mathcal{C} \mathcal{P}$-violating couplings to the third generation quarks. Final conclusions are drawn in Section 5 .

\section{Outline of the calculation and matrix elements}

The production of the $\mathcal{C P}$-odd Higgs boson $A$ in association with two jets, at order $\alpha_{s}^{4}$, proceeds in analogy to the production of the $\mathcal{C} \mathcal{P}$-even Higgs boson $H_{S M}$ of the SM. The $H_{S M} j j$ production processes with full heavy quark mass effects were considered in Ref. [1], and we here closely follow the framework and the notation introduced there. We consider the production subprocesses

$$
q q \rightarrow q q A, \quad q Q \rightarrow q Q A, \quad q g \rightarrow q g A, \quad g g \rightarrow g g A,
$$

and all crossing-related processes. Here the first two entries denote scattering of identical and non-identical quark flavors. The Higgs boson $A$ is produced by massive quark loops, for which only the third quark generation is taken into account. Furthermore, within the MSSM, massive squark loops can safely be neglected, because their contribution sums to zero at amplitude level in the production of a $\mathcal{C P}$-odd Higgs boson [11]. In the 2HDM, up- and down-type quark Yukawa couplings depend on the ratio of vacuum expectation values, $\tan \beta=v_{u} / v_{d}$, via the relations

\section{2HDM of type I:}

$$
\tilde{y}_{A, u u}^{\mathrm{I}}=\frac{\cot \beta}{v} m_{u} \quad \text { and } \quad \tilde{y}_{A, d d}^{\mathrm{I}}=-\frac{\cot \beta}{v} m_{d},
$$

\section{2HDM of type II (MSSM):}

$$
\tilde{y}_{A, u u}^{\mathrm{II}}=-\frac{\cot \beta}{v} m_{u} \quad \text { and } \quad \tilde{y}_{A, d d}^{\mathrm{II}}=-\frac{\tan \beta}{v} m_{d} .
$$

In the 2HDM of type I, Yukawa couplings for up-type and down-type quarks are suppressed equally at large $\tan \beta$ compared to the $2 \mathrm{HDM}$ of type II, where only the up-type Yukawa 
coupling is suppressed but the down-type Yukawa coupling is enhanced. Due to this enhancement, loops with bottom quarks can also provide significant contributions to cross sections.

In the calculation of the subprocesses listed in (2.1), three different loop topologies appear: the triangle-, box-, and pentagon diagrams of Fig. 1. The contributing Feynman

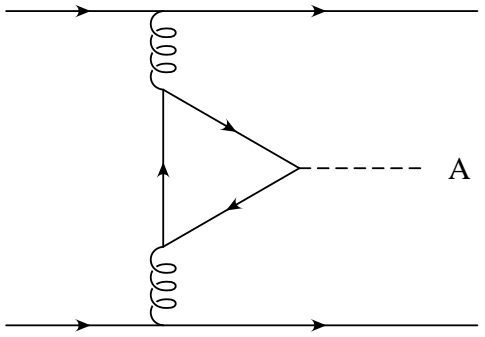

(a)

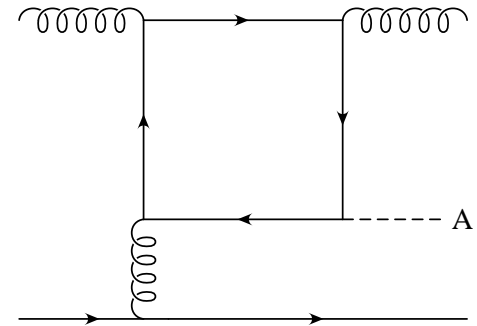

(b)

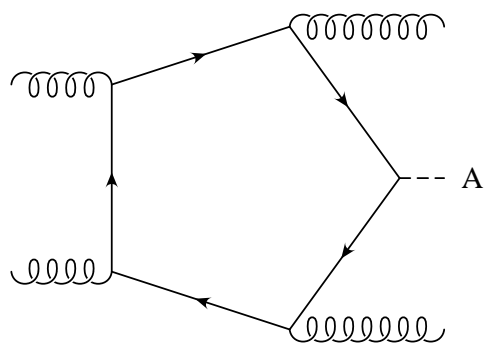

(c)

Figure 1: Examples of Feynman graphs contributing to $A+2$ jet production via gluon fusion.

diagrams can be easily built from the simpler QCD dijet processes at leading order. One needs to insert the Higgs-gluon triangles into the gluon propagators of the $2 \rightarrow 2$ tree-level diagrams in all possible ways or one replaces a triple gluon or four gluon vertex by box or pentagon graphs in all possible ways. Charge-conjugation related diagrams, where the loop momentum is running clockwise and counter-clockwise, can be counted as one by exploiting Furry's theorem [12]. This effectively reduces the number of diagrams by a factor of two. Furthermore all diagrams are UV-convergent and, due to the finite quark mass in the loops, also IR-convergent. All coupling constants and loop factors which appear can conveniently be absorbed into an overall factor

$$
F_{f}=4 m_{f} h_{f} \frac{g_{s}^{4}}{16 \pi^{2}}=4 m_{f} h_{f} \alpha_{s}^{2},
$$

where $f=b, t$ labels the heavy quark flavor of a particular loop. In the following, we use the MSSM couplings of Eq. (2.3), i.e. we set $h_{t}=\cot \beta m_{t} / v$ and $h_{b}=\tan \beta m_{b} / v$. By pulling out a loop factor $4 m_{f} / 16 \pi^{2}$, we anticipate that the Dirac trace of all loops requires a quark mass insertion to compensate the helicity flip induced by the $\Phi f \bar{f}$ coupling.

\subsection{Subprocesses $q Q \rightarrow q Q A$ and $q q \rightarrow q q A$}

The subprocess $q Q \rightarrow q Q A$, depicted in Fig. 1(a), is the simplest contribution to $A+2$ jet production. Following Ref. [1, the amplitude for different flavors can be written as

$$
\mathcal{A}^{q Q}=\sum_{f=t, b} F_{f}^{q Q} J_{21}^{\mu_{1}} J_{43}^{\mu_{2}} T_{\mu_{1} \mu_{2}}\left(q_{1}, q_{2}, m_{f}\right) t_{i_{2} i_{1}}^{a} t_{i_{4} i_{3}}^{a}=\mathcal{A}_{2143}^{q Q} t_{i_{2} i_{1}}^{a} t_{i_{4} i_{3}}^{a} .
$$

Using the notation and formalism for the spinor algebra of [13, the external quark lines can be expressed by effective quark currents $J_{21}^{\mu_{1}}$ and $J_{43}^{\mu_{2}}$ as given in [1]. The triangle 
tensor $T^{\mu_{1} \mu_{2}}\left(q_{1}, q_{2}, m_{f}\right)$ (see Appendix Fig. 9) has the simple form

$$
T^{\mu_{1} \mu_{2}}\left(q_{1}, q_{2}, m_{f}\right)=\varepsilon^{\mu_{1} \mu_{2} q_{1} q_{2}} C_{0}\left(q_{1}, q_{2}, m_{f}\right) .
$$

Here, $C_{0}$ denotes the scalar three-point function and $\varepsilon^{\mu_{1} \mu_{2} q_{1} q_{2}}$ is the totally anti-symmetric tensor (Levi-Civita symbol) in four dimensions contracted with attached gluon momenta $q_{1}$ and $q_{2}$. The $t_{i j}^{a}=\lambda_{i j}^{a} / 2$ are color generators in the fundamental representation of $\mathrm{SU}(N), N=3$ and the overall factor

$$
F_{f}^{q Q}=S_{1} S_{2} S_{3} S_{4} 4 \sqrt{\bar{p}_{1}^{0} \bar{p}_{2}^{0} \bar{p}_{3}^{0} \bar{p}_{4}^{0}} F_{f}
$$

includes normalization factors of external quark spinors. Here, the $\bar{p}_{i}$ denote physical momenta describing phase space and the wave functions of fermions and bosons while $p_{i}$ is used for momenta appearing in the momentum flow in Feynman diagrams. Both sets of momenta are related by the sign factors $S_{i}[13$

$$
p_{i}=S_{i} \bar{p}_{i}
$$

with $S_{i}=+1$ for fermions and $S_{i}=-1$ for anti-fermions. The factor $F_{f}$ is given in Eq. (2.4). For identical quark flavors, one has to keep in mind Pauli interference

$$
\mathcal{A}^{q q}=\mathcal{A}_{2143}^{q q} t_{i_{2} i_{1}}^{a} t_{i_{4} i_{3}}^{a}-\mathcal{A}_{4123}^{q q} t_{i_{4} i_{1}}^{a} t_{i_{2} i_{3}}^{a} .
$$

The squared amplitude, summed over initial- and final-particle color, becomes

$$
\sum_{\text {color }}\left|\mathcal{A}^{q q}\right|^{2}=\left(\left|\mathcal{A}_{2143}\right|^{2}+\left|\mathcal{A}_{4123}\right|^{2}\right) \frac{N^{2}-1}{4}+2 \operatorname{Re}\left(\mathcal{A}_{2143} \mathcal{A}_{4123}^{*}\right) \frac{N^{2}-1}{4 N} .
$$

\section{$2.2 \quad$ Subprocess $q g \rightarrow q g A$}

Polarization vectors of external gluon lines with a triangle insertion can be expressed by effective polarization vectors

$$
e_{i A}^{\mu}\left(m_{f}\right)=\varepsilon^{\mu \epsilon_{i} q_{i} P} \frac{1}{\left(q_{i}+P\right)^{2}} C_{0}\left(q_{i},-\left(q_{i}+P\right), m_{f}\right),
$$

which replace the polarization vectors $\epsilon_{i}^{\mu}$ of the underlying $2 \rightarrow 2$ process for gluons $i=1,2$. Here $q_{i}$ is the external gluon momentum while $P$ denotes the momentum of the Higgs boson. The expression for the amplitude of graphs with a triangle insertion adjacent to a three-gluon vertex differs slightly from that in [1] due to the emergence of the Levi-Civita symbol

$$
\begin{aligned}
\mathcal{A}_{\mathrm{tri}}^{q g}=\sum_{f} F_{f}^{q g}[ & \left.t^{a_{1}}, t^{a_{2}}\right]_{i_{1} i_{2}}\left\{2\left[e_{1 A} \cdot \epsilon_{2} J_{21} \cdot q_{2}-e_{1 A} \cdot J_{21} \epsilon_{2} \cdot\left(p_{2}-p_{1}\right)-e_{1 A} \cdot q_{2} J_{21} \cdot \epsilon_{2}\right]\right. \\
& -2\left[e_{2 A} \cdot \epsilon_{1} J_{21} \cdot q_{1}-e_{2 A} \cdot q_{1} J_{21} \cdot \epsilon_{1}-e_{2 A} \cdot J_{21} \epsilon_{1} \cdot\left(p_{2}-p_{1}\right)\right] \\
& +2 \varepsilon_{\mu_{1} \mu_{2} \mu_{3} \mu_{4}} J_{21}^{\mu_{2}}\left[\epsilon_{1} \cdot \epsilon_{2} q_{1}^{\mu_{3}} q_{2}^{\mu_{1}}\left(p_{2}-p_{1}\right)^{\mu_{4}}+\left(\epsilon_{2} \cdot q_{1} \epsilon_{1}^{\mu_{1}}-\epsilon_{1} \cdot q_{2} \epsilon_{2}^{\mu_{1}}\right)\right. \\
& \left.\left.\times\left(q_{1}+q_{2}\right)^{\mu_{3}}\left(p_{2}-p_{1}\right)^{\mu_{4}}\right] \frac{C_{0}\left(p_{2}-p_{1}, q_{1}+q_{2}, m_{f}\right)}{\left(q_{1}+q_{2}\right)^{2}}\right\} .
\end{aligned}
$$


Further expressions for amplitudes of graphs with a triangle insertion can be taken from [1] replacing $e_{i H}^{\mu}$ by $e_{i A}^{\mu}\left(m_{f}\right)$. The tensor structure of the box diagram in Fig. 1(b) is given by $\bar{B}_{\mu_{1} \mu_{2} \mu_{3}}\left(q_{1}, q_{2}, q_{3}, m_{f}\right)$, which is shown pictorially in Fig. 10 and given explicitly in Appendix B. Finally the color structure of the $q g \rightarrow q g A$ amplitude is given by [1]

$$
\mathcal{A}^{q g}=\left(t^{a_{1}} t^{a_{2}}\right)_{i_{2} i_{1}} \mathcal{A}_{12}^{q g}+\left(t^{a_{2}} t^{a_{1}}\right)_{i_{2} i_{1}} \mathcal{A}_{21}^{q g} \quad \text { with } \quad \mathcal{A}^{q g}=\sum_{f} \mathcal{A}_{f}^{q g} .
$$

The indices 12 and 21 label amplitudes with interchanged external gluons. Thus, the resulting color-summed squared amplitude takes the form

$$
\sum_{\text {color }}\left|\mathcal{A}^{q g}\right|^{2}=\left(\left|\mathcal{A}_{12}^{q g}\right|^{2}+\left|\mathcal{A}_{21}^{q g}\right|^{2}\right) \frac{\left(N^{2}-1\right)^{2}}{4 N}-2 \operatorname{Re}\left[\mathcal{A}_{12}^{q g}\left(\mathcal{A}_{21}^{q g}\right)^{*}\right] \frac{N^{2}-1}{4 N} .
$$

\subsection{Subprocess $g g \rightarrow g g A$}

After inserting suitable loop topologies and application of Furry's theorem, this process contains 19 graphs with triangle insertions, 18 box contributions and 12 pentagon diagrams. The pentagon diagrams Fig. 1(c) enter via the $P^{\mu_{1} \mu_{2} \mu_{3} \mu_{4}}$ tensor (see Appendix Fig. 11). Full expressions and diagrams can be looked up in [16]. The contributing color structures to the process $g g \rightarrow g g A$ can be expressed by the real-valued color coefficients $c_{i}$ defined in [1]

$$
\begin{aligned}
& c_{1}=\operatorname{tr}\left[t^{a_{1}} t^{a_{2}} t^{a_{3}} t^{a_{4}}\right]+\operatorname{tr}\left[t^{a_{1}} t^{a_{4}} t^{a_{3}} t^{a_{2}}\right], \\
& c_{2}=\operatorname{tr}\left[t^{a_{1}} t^{a_{3}} t^{a_{4}} t^{a_{2}}\right]+\operatorname{tr}\left[t^{a_{1}} t^{a_{2}} t^{a_{4}} t^{a_{3}}\right], \\
& c_{3}=\operatorname{tr}\left[t^{a_{1}} t^{a_{4}} t^{a_{2}} t^{a_{3}}\right]+\operatorname{tr}\left[t^{a_{1}} t^{a_{3}} t^{a_{2}} t^{a_{4}}\right] .
\end{aligned}
$$

Evaluation of the color traces yields

$$
\begin{aligned}
& c_{1}=\frac{1}{4}\left(\frac{2}{N} \delta^{a_{1} a_{2}} \delta^{a_{3} a_{4}}+d^{a_{1} a_{2} l} d^{a_{3} a_{4} l}-f^{a_{1} a_{2} l} f^{a_{3} a_{4} l}\right), \\
& c_{2}=\frac{1}{4}\left(\frac{2}{N} \delta^{a_{1} a_{3}} \delta^{a_{4} a_{2}}+d^{a_{1} a_{3} l} d^{a_{4} a_{2} l}-f^{a_{1} a_{3} l} f^{a_{4} a_{2} l}\right), \\
& c_{3}=\frac{1}{4}\left(\frac{2}{N} \delta^{a_{1} a_{4}} \delta^{a_{2} a_{3}}+d^{a_{1} a_{4} l} d^{a_{2} a_{3} l}-f^{a_{1} a_{4} l} f^{a_{2} a_{3} l}\right) .
\end{aligned}
$$

In terms of these color coefficients, the complete amplitude for $g g \rightarrow g g A$ can be decomposed into three separately gauge invariant sub-amplitudes

$$
\mathcal{A}^{g g}=\sum_{i=1}^{3} c_{i} \sum_{f} \mathcal{A}_{i, f}^{g g} .
$$

The sum over colors of the external gluons for the squared amplitude becomes

$$
\sum_{\text {color }}\left|\mathcal{A}^{g g}\right|^{2}=\sum_{i, j=1}^{3} \mathcal{A}_{i}^{g g}\left(\mathcal{A}_{j}^{g g}\right)^{*} \sum_{\text {color }} c_{i} c_{j},
$$


where the color factors are given by

$$
\begin{aligned}
& \mathcal{C}_{1} \equiv \sum_{\text {color }} c_{i} c_{i}=\frac{\left(N^{2}-1\right)\left(N^{4}-2 N^{2}+6\right)}{8 N^{2}},(\text { no sum. over } i) \\
& \mathcal{C}_{2} \equiv \sum_{\text {color }} c_{i} c_{j}=\frac{\left(N^{2}-1\right)\left(3-N^{2}\right)}{4 N^{2}}, \quad i \neq j .
\end{aligned}
$$

Thus, one finally gets

$$
\sum_{\text {color }}\left|\mathcal{A}^{g g}\right|^{2}=\mathcal{C}_{1} \sum_{i=1}^{3}\left|\mathcal{A}_{i}^{g g}\right|^{2}+\mathcal{C}_{2} \sum_{i, j=1 ; i \neq j}^{3} \mathcal{A}_{i}^{g g}\left(\mathcal{A}_{j}^{g g}\right)^{*} .
$$

\section{Numerical implementation}

Analytic expressions for the amplitudes of the previous chapter were implemented in the Fortran program VBFNLO [10, 17]. The tensor reduction of the loop contributions up to boxes is performed via Passarino-Veltman reduction [14]. Additionally we avoid the explicit calculation of the inverse of the Gram matrix by solving system of linear equations, which is numerically more stable close to the singular points. For pentagons, we use the Denner-Dittmaier algorithm [18] which avoids the inversion of small Gram determinants, in particular for planar configurations of the Higgs and the two final state partons. The program was numerically tested in several ways. Besides usual gauge-invariance and Lorentz-invariance tests of the amplitudes, the different topologies were checked separately. The contraction of a triangle-tensor $T_{\mu_{1} \mu_{2}}\left(q_{1}, q_{2}, m_{f}\right)$ with gluon momentum $q_{i}^{\mu}$ has to vanish due to total antisymmetry of the Levi-Civita symbol

$$
q_{1}^{\mu_{1}} T_{\mu_{1} \mu_{2}}\left(q_{1}, q_{2}, m_{f}\right)=q_{2}^{\mu_{2}} T_{\mu_{1} \mu_{2}}\left(q_{1}, q_{2}, m_{f}\right)=0 .
$$

Contracting with external gluon momenta, the tensor expressions of boxes and pentagons reduce to differences of triangles and boxes, respectively. With the tensor integrals as defined in the Appendix, the Ward identities for the boxes read

$$
\begin{aligned}
& q_{1}^{\mu_{1}} B_{\mu_{1} \mu_{2} \mu_{3}}\left(q_{1}, q_{2}, q_{3}, m_{f}\right)=T_{\mu_{2} \mu_{3}}\left(q_{12}, q_{3}, m_{f}\right)-T_{\mu_{2} \mu_{3}}\left(q_{2}, q_{3}, m_{f}\right), \\
& q_{2}^{\mu_{2}} B_{\mu_{1} \mu_{2} \mu_{3}}\left(q_{1}, q_{2}, q_{3}, m_{f}\right)=T_{\mu_{1} \mu_{3}}\left(q_{1}, q_{23}, m_{f}\right)-T_{\mu_{1} \mu_{3}}\left(q_{12}, q_{3}, m_{f}\right) \\
& q_{3}^{\mu_{3}} B_{\mu_{1} \mu_{2} \mu_{3}}\left(q_{1}, q_{2}, q_{3}, m_{f}\right)=T_{\mu_{1} \mu_{2}}\left(q_{1}, q_{2}, m_{f}\right)-T_{\mu_{1} \mu_{2}}\left(q_{1}, q_{23}, m_{f}\right)
\end{aligned}
$$

where the abbreviation $q_{i j}=q_{i}+q_{j}$ has been used. Similarly, for the pentagons one finds

$$
\begin{aligned}
& q_{1}^{\mu_{1}} P_{\mu_{1} \mu_{2} \mu_{3} \mu_{4}}\left(q_{1}, q_{2}, q_{3}, q_{4}, m_{f}\right)=B_{\mu_{2} \mu_{3} \mu_{4}}\left(q_{12}, q_{3}, q_{4}, m_{f}\right)-B_{\mu_{2} \mu_{3} \mu_{4}}\left(q_{2}, q_{3}, q_{4}, m_{f}\right), \\
& q_{2}^{\mu_{2}} P_{\mu_{1} \mu_{2} \mu_{3} \mu_{4}}\left(q_{1}, q_{2}, q_{3}, q_{4}, m_{f}\right)=B_{\mu_{1} \mu_{3} \mu_{4}}\left(q_{1}, q_{23}, q_{4}, m_{f}\right)-B_{\mu_{1} \mu_{3} \mu_{4}}\left(q_{12}, q_{3}, q_{4}, m_{f}\right), \\
& q_{3}^{\mu_{3}} P_{\mu_{1} \mu_{2} \mu_{3} \mu_{4}}\left(q_{1}, q_{2}, q_{3}, q_{4}, m_{f}\right)=B_{\mu_{1} \mu_{2} \mu_{4}}\left(q_{1}, q_{2}, q_{34}, m_{f}\right)-B_{\mu_{1} \mu_{2} \mu_{4}}\left(q_{1}, q_{23}, q_{4}, m_{f}\right), \\
& q_{4}^{\mu_{4}} P_{\mu_{1} \mu_{2} \mu_{3} \mu_{4}}\left(q_{1}, q_{2}, q_{3}, q_{4}, m_{f}\right)=B_{\mu_{1} \mu_{2} \mu_{3}}\left(q_{1}, q_{2}, q_{3}, m_{f}\right)-B_{\mu_{1} \mu_{2} \mu_{3}}\left(q_{1}, q_{2}, q_{34}, m_{f}\right) .
\end{aligned}
$$


These relationships were tested numerically and they, typically, are satisfied at the $10^{-9}$ level when using Denner-Dittmaier reduction for the tensor integrals. In addition, one can perform a QED-check for the pentagons. Replacing gluons by photons and considering the process $\gamma \gamma \rightarrow \gamma \gamma A$, diagrams with three- and four-gluon-vertices vanish, because these structures are not available in an Abelian theory. The amplitude is simply given by the sum of all pentagon graphs, without color factors. When contracting with an external gauge boson momentum one obtains zero, since boxes are not allowed for photons, by Furry's theorem. Our amplitudes pass this test as well.

To check the full scattering amplitudes, one can make use of the heavy-top effective Lagrangian for a SM strength Yukawa coupling,

$$
\mathcal{L}_{\text {eff }}^{A}=\frac{\alpha_{s}}{8 \pi v} G_{\mu_{1} \mu_{2}}^{a} \tilde{G}^{a \mu_{1} \mu_{2}} A \quad \text { with } \quad \tilde{G}^{a \mu_{1} \mu_{2}}=\frac{1}{2} \epsilon^{\mu_{1} \mu_{2} \mu_{3} \mu_{4}} G_{\mu_{3} \mu_{4}}^{a} .
$$

As $m_{t}$ becomes large, the results calculated with full fermion loops must approach the approximate ones derived from the effective Lagrangian. This check was performed with $m_{t}=5000 \mathrm{GeV}$, and cross sections agreed well for Higgs boson masses in the range 100 $\mathrm{GeV}<m_{A}<1000 \mathrm{GeV}$. In production runs, we put a cut in the routines for the determination of the tensor integral coefficients of the $\mathrm{C}$ and $\mathrm{D}$ functions such that the complete amplitude is set to zero when small Gram determinants appear. We have checked that the result and plots do not depend on this cut for a broad range of values. Finally, the amplitudes for all three subprocesses were recalculated using the FeynCalc/FormCalc framework [15]. For a selection of randomly generated phase space points, the two independent calculations yield agreement at least at the $10^{-6}$ level.

\section{Applications to LHC physics}

The numerical analysis of the $\Phi+2$ jet cross section was performed with a parton level Monte Carlo program in the VBFNLO framework [17], using the CTEQ6L1 [19] set for parton-distribution functions. In order to prevent soft or collinear divergencies in the cross sections, a minimal set of acceptance cuts has to be introduced. Following Ref. [1], we impose

$$
p_{T j}>20 \mathrm{GeV}, \quad\left|\eta_{j}\right|<4.5, \quad R_{j j}>0.6,
$$

where $p_{T j}$ is the transverse momentum of a final state parton and $R_{j j}$ describes the separation of the two partons in the pseudo-rapidity versus azimuthal-angle plane

$$
R_{j j}=\sqrt{\Delta \eta_{j j}^{2}+\phi_{j j}^{2}}
$$

with $\Delta \eta_{j j}=\left|\eta_{j 1}-\eta_{j 2}\right|$ and $\phi_{j j}=\phi_{j 1}-\phi_{j 2}$. These cuts anticipate LHC detector capabilities and jet finding algorithms and will be called "inclusive cuts" (IC) in the following. Unless specified otherwise, the factorization scale is set to

$$
\mu_{f}=\sqrt{p_{T 1} p_{T 2}}
$$




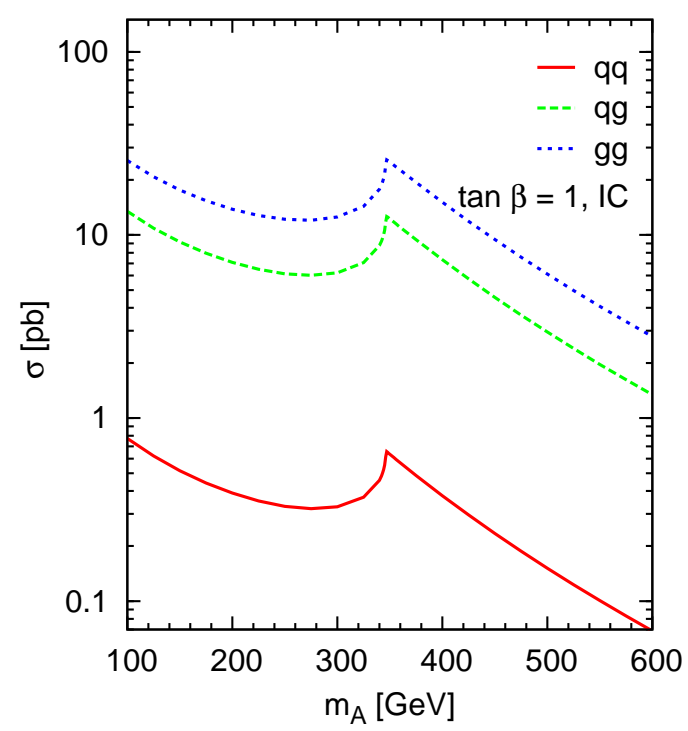

Figure 2: A+2 jet cross section of the individual contributions of the subprocesses quark-quark, quark-gluon and gluon-gluon scattering for $\tan \beta=1$ as a function of the pseudo-scalar Higgs boson mass, $m_{A}$. Here, the inclusive cuts (IC) of Eq. (4.1) were applied.

while the renormalization scale is fixed by setting [1]

$$
\alpha_{s}^{4}\left(\mu_{R}\right)=\alpha_{s}\left(p_{T 1}\right) \alpha_{s}\left(p_{T 2}\right) \alpha_{s}^{2}\left(m_{A}\right) .
$$

We use one-loop $\alpha_{s}$ running with $\alpha_{s}\left(M_{Z}\right)=0.13$. All our results below contain the contributions from the full top- and bottom-quark loops. For the top-quark mass we use $m_{t}=173.1 \mathrm{GeV}$. In the case of bottom-loops, running Yukawa coupling and propagator mass are taken into account, with the Higgs-mass as reference scale. Within the Higgsmass range of 100 to $600 \mathrm{GeV}$, the bottom-quark mass is 33 to $42 \%$ smaller than the pole mass of $4.855 \mathrm{GeV}$. The evolution of $m_{b}$ up to a reference scale $\mu$ can be expressed as

$$
\bar{m}_{b}(\mu)=\bar{m}_{b}\left(m_{b}\right) \frac{c\left[\alpha_{s}(\mu) / \pi\right]}{c\left[\alpha_{s}\left(m_{b}\right) / \pi\right]}
$$

with $\bar{m}_{b}\left(m_{b}\right)=4.2 \mathrm{GeV}$, as derived from the relation between pole mass and MS-bar mass. For the coefficient function $c$, the five flavor approximation [20, 21] within the mass range $m_{b}<\mu<m_{t}$,

$$
c(x)=\left(\frac{23}{6} x\right)^{\frac{12}{23}}\left[1+1.17549 x+1.50071 x^{2}+0.172478 x^{3}\right],
$$

is used. Further evolution of $\bar{m}_{b}$ to a renormalization scale $\mu>m_{t}$ can be performed safely within the five flavor approximation, because the deviation to the six flavor scheme is less than $1 \%$ for $\mu<600 \mathrm{GeV}$.

Contributions of individual subprocess categories to the total cross section for $\tan \beta=$ 1 are shown in Fig. 2 as a function of the Higgs boson mass, $m_{A}$. Here, the minimal cuts 

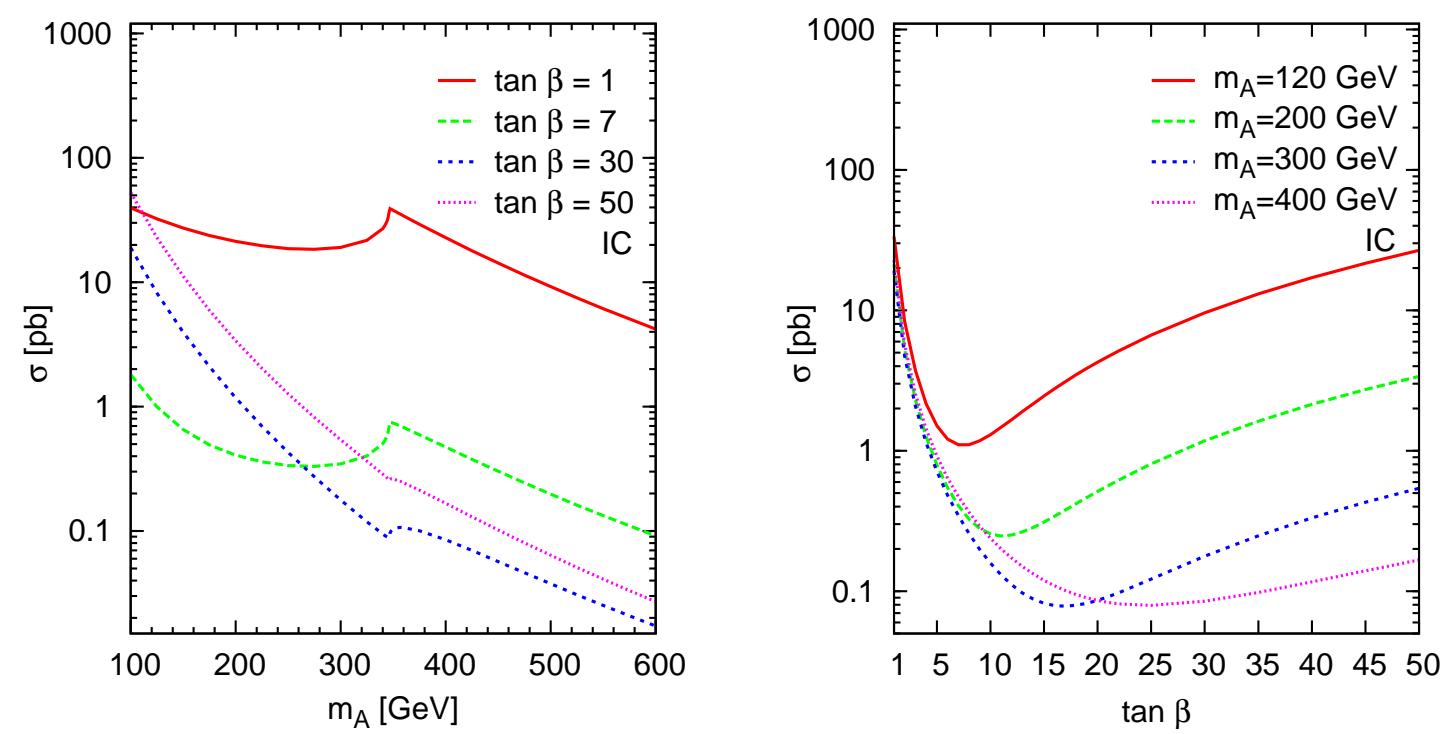

Figure 3: $A+2$ jet cross section as a function of the pseudo-scalar Higgs boson mass, $m_{A}$ for different values of $\tan \beta$. The inclusive cuts (IC) of Eq. (4.1) are applied

of Eq. (4.1) were used. The cross sections for processes involving gluons (quark-gluon or gluon-gluon amplitudes) exceed the quark-quark scattering contributions by more than one order of magnitude. The $m_{A}$ dependence of the full cross section, with top- and bottom-quark interference, is given in the left panel of Fig. 3 for a selection of $\tan \beta$ values. For small $\tan \beta$, amplitudes with a top-quark loop dominate over bottom-quark loop mediated contributions. The striking peak arises due to threshold enhancement near $m_{A} \approx 2 m_{t}$, whereas for bottom-quark loop dominated processes the peak would appear well below the Higgs mass range shown.

For low $m_{A}$, the minimal cross section is obtained near $\tan \beta \approx 7$, when $h_{t} \approx h_{b}$ (see Eq. (2.3) $)$ and both Yukawa couplings are suppressed compared to $h_{t}^{S M}$. For large $\tan \beta$, e.g. $\tan \beta=50$ in Fig. 3 (a), the bottom-quark loops dominate. However, they lead to a much more rapid decrease of the cross section with rising $m_{A}$ because the suppression scale of the loops is now set by the heavy Higgs boson mass instead of the quark mass. The reduced importance of the bottom-quark loops at large $m_{A}$ implies that equality of the top and bottom contributions and, thereby, the minimum of the production cross section is reached at increasingly larger $\tan \beta$ as $m_{A}$ is increased. This effect is demonstrated in the right panel of Fig. 3 .

The $\gamma_{5}$-matrix in the Dirac-trace of the quark loops leads to a new tensor structure and to a normalization of the loops that, for equal Yukawa couplings, induces a $(3 / 2)^{2}=2.25$ times larger $A j j$ than $H j j$ cross section. This enhancement is shown in Fig. 4 and is also apparent in the effective Lagrangian of Eq. (1.1), where the coefficient of the $\mathcal{C P}$-odd Agg coupling exceeds that of the $\mathrm{Hgg}$ coupling by a factor $3 / 2$. This effective Lagrangian provides a good approximation to the total $\Phi j j$ cross sections up to Higgs- 


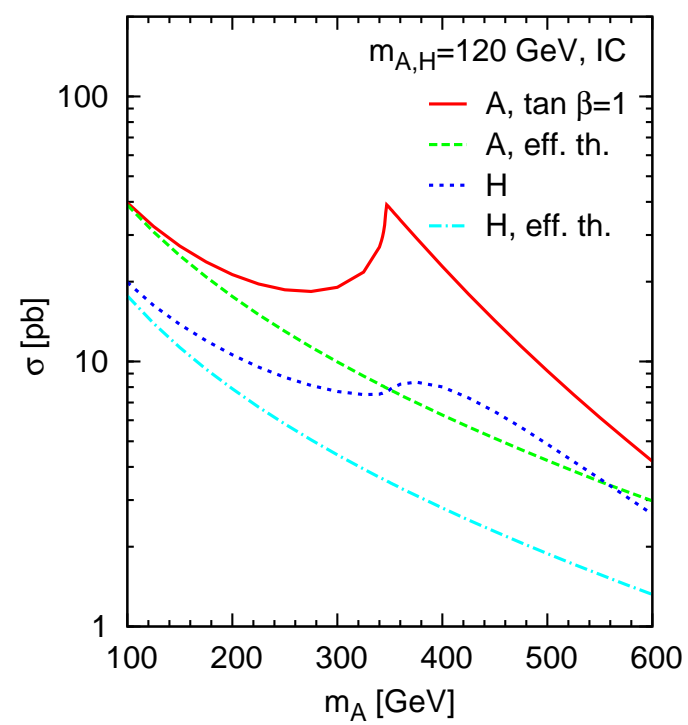

Figure 4: Comparison of cross sections for the $\mathcal{C P}$-odd and $\mathcal{C P}$-even Higgs for the full loop calculation and within the effective theory. The inclusive cuts (IC) of Eq. (4.1) are applied.

masses of $\approx 160 \mathrm{GeV}$ and for small transverse momenta, $p_{T j} \lesssim m_{t}$. In this region, the effective Lagrangian approximation can be used as a numerically fast alternative for phenomenological studies [1].

The smaller quark mass in the bottom loops also has a pronounced effect on the transverse momentum distribution of the accompanying jets: for $p_{T j} \gtrsim m_{b}$ the large scale of the kinematic invariants leads to an additional suppression of the bottom induced subamplitudes compared to the heavy quark effective theory. This effect is clearly visible in Figs. 5 and [6, where the transverse-momentum distributions of the softer and the harder of the two jets are shown for pseudoscalar Higgs masses $m_{A}=120,200$ and $400 \mathrm{GeV}$ for $\tan \beta=1,7,30$. For modest Higgs mass values, both distributions fall more steeply for large $\tan \beta$. At large values of $m_{A}$, the Higgs boson mass sets the scale for the fermion loops which, in the $m_{A}=400 \mathrm{GeV}$ panels of Figs. 5 and 6 , leads to $p_{T}$ distributions which are approximately equal for the top- or bottom-quark dominated loops.

The azimuthal angle between the more forward and the more backward of the two tagging jets, $\phi_{j j}=\phi_{j F}-\phi_{j B}$, provides a sensitive probe for the $\mathcal{C} \mathcal{P}$-character of the Higgs couplings to the quarks [22, 24, 26]. As shown in the left panel of Fig. 8 for a heavy quark in the loop, the $\mathcal{C P}$-even $H q q$ coupling produces a minimum for $\phi_{j j}= \pm 90$ degrees while a $\gamma_{5}$-induced $\mathcal{C} \mathcal{P}$-odd $A q q$ coupling leads to minima at $\phi_{j j}=0$ and \pm 180 degrees. The softening effects observed for the jet transverse momentum distribution then raise the question, to what extent the jet azimuthal angle correlations of the effective theory will get modified when bottom quark loops dominate.

For the set of pseudoscalar Higgs masses and $\tan \beta$ values mentioned above, predictions for the normalized $\phi_{j j}$-distributions are shown in Fig. 7. The calculation was carried out 

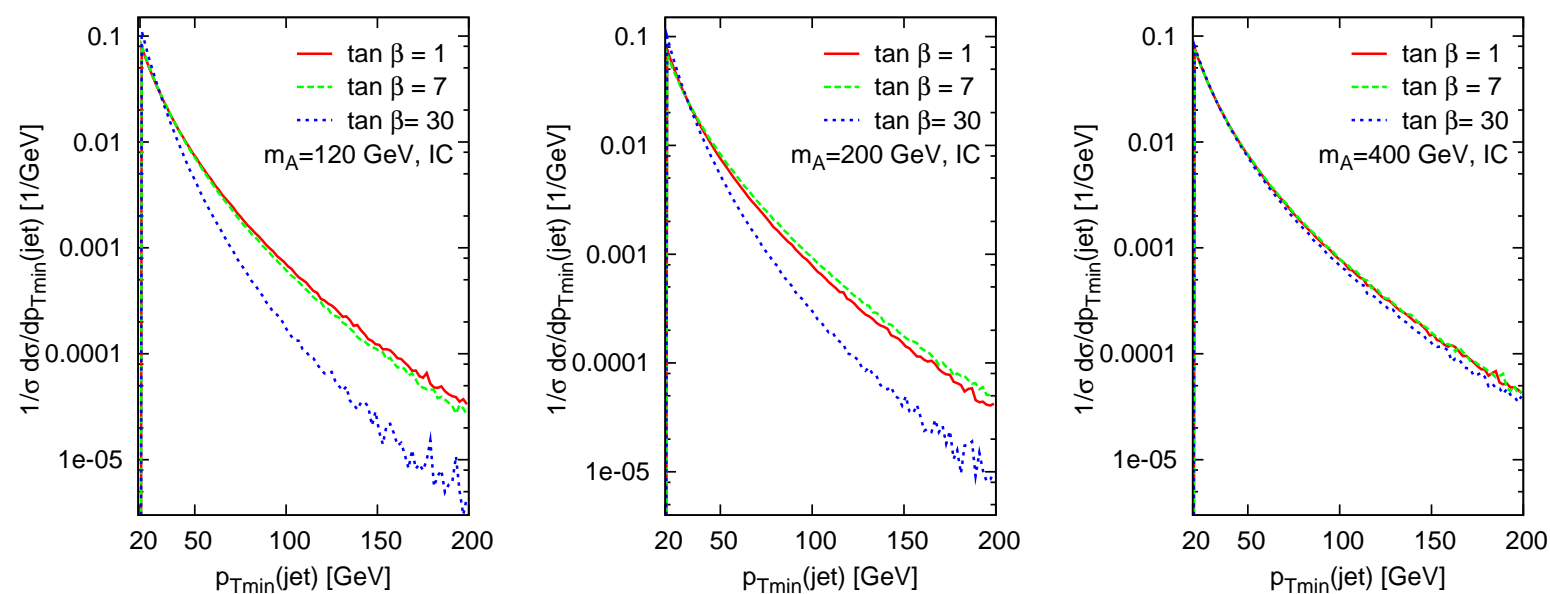

Figure 5: Normalized transverse-momentum distributions of the softer jet in Ajj production at the LHC, for different $\tan \beta$ and Higgs-mass values. The inclusive selection cuts of Eq. (4.1) are applied.
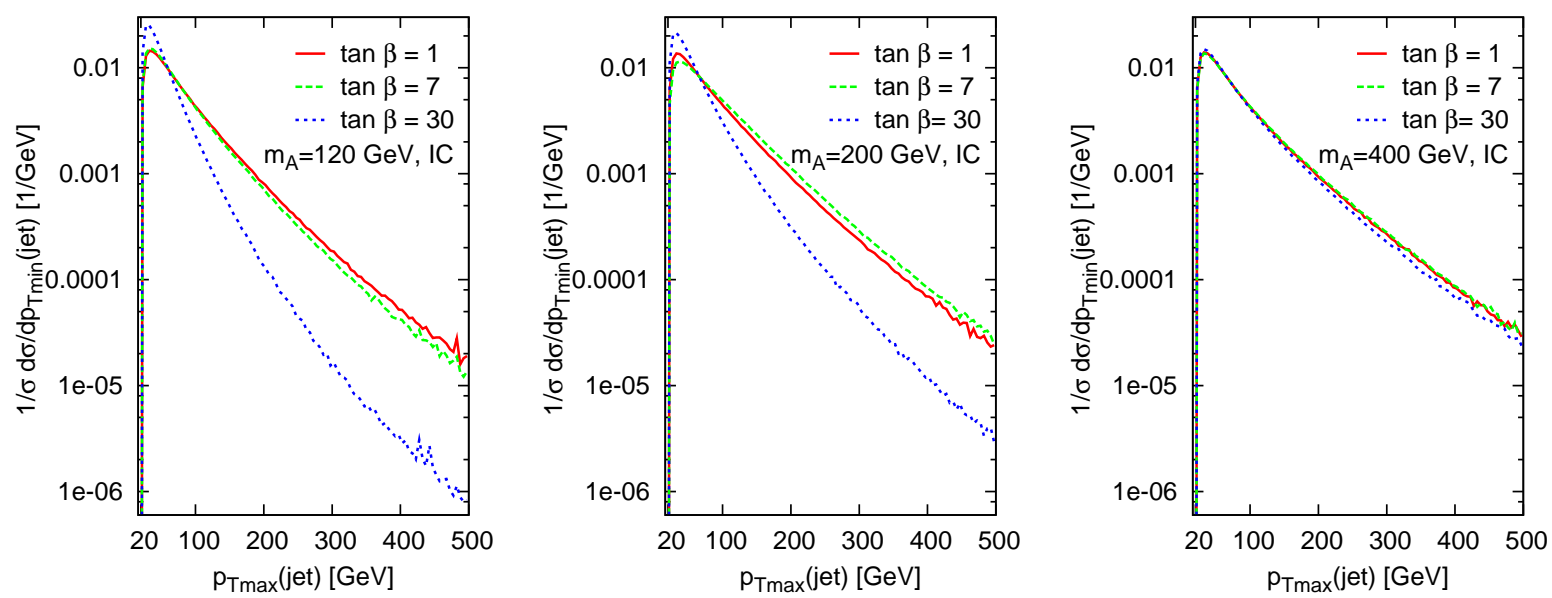

Figure 6: Normalized transverse-momentum distributions of the harder jet in Ajj production at the LHC for different $\tan \beta$ and Higgs-mass values. The inclusive selection cuts of Eq. (4.1) are applied.

with a modified set of cuts, however, which was shown in Ref. [25] to lead to a better sensitivity to the $\mathcal{C} \mathcal{P}$-structure of the Higgs couplings than the inclusive cuts. In Fig. 7 , we use

$$
p_{T j}>30 \mathrm{GeV}, \quad\left|\eta_{j}\right|<4.5, \quad R_{j j}>0.6, \quad \Delta \eta_{j j}=\left|\eta_{j 1}-\eta_{j 2}\right|>3,
$$

which we call ICphi cuts in the following. One finds that the characteristic structure of the $\phi_{j j}$ distribution, dips at $\phi_{j j}=0$ and $\phi_{j j}= \pm 180$ degrees, remains for bottom quark dominated $A j j$ production, albeit at a quantitatively reduced level for $m_{A}>2 m_{q}$. For a relatively light pseudoscalar Higgs boson and large $\tan \beta$, the softer transverse momentum distribution of the Higgs leads to kinematical distortions of the $\phi_{j j}$ distribution: at $\phi_{j j} \approx 0$ 

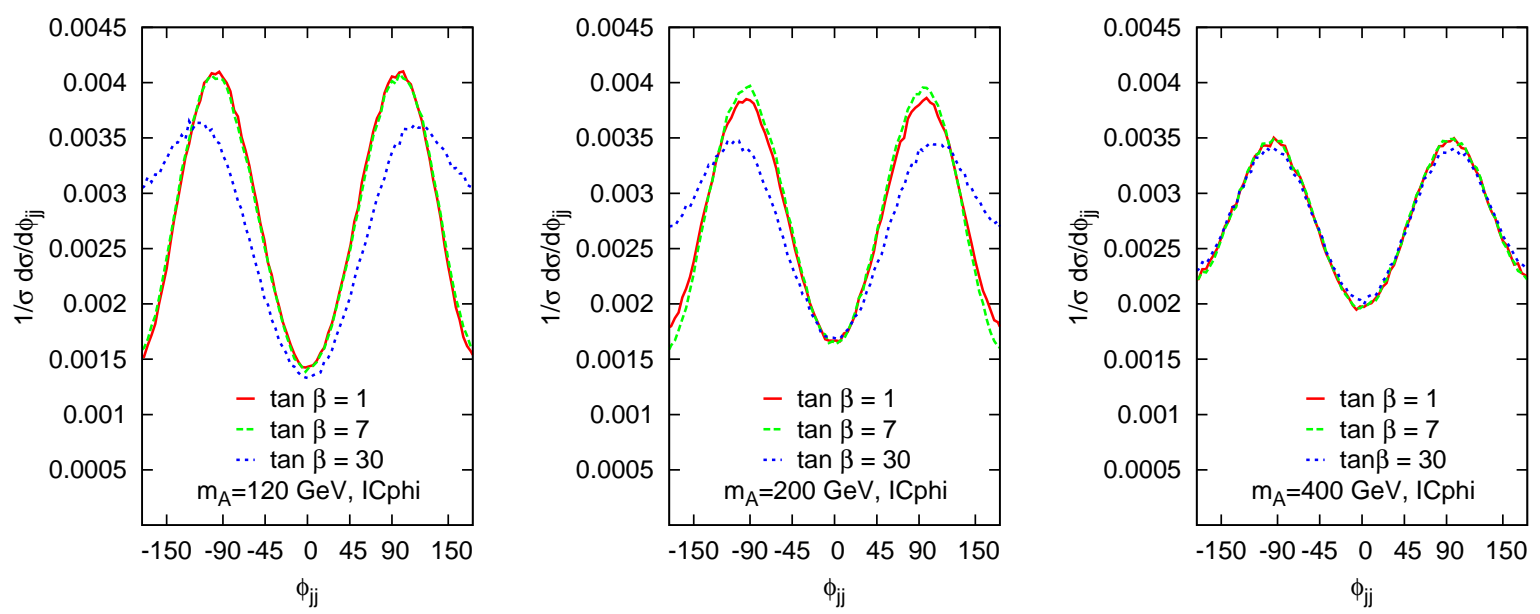

Figure 7: Distribution in the azimuthal-angle between the two final state jets for $\mathcal{C P}$-odd Higgs boson production at different Higgs-masses and $\tan \beta$ values. The ICphi set of acceptance cuts (see Eq. (4.7)) is used for pp collisions at $14 \mathrm{TeV}$.

the Higgs recoils against two jets and hence must have $p_{T H}>60 \mathrm{GeV}$, and this high $p_{T^{-}}$ scale leads to an additional suppression as compared to the $\phi_{j j} \approx \pm 180$ degree case where transverse momentum balancing of the jets does allow $p_{T H}=0$.

For the effective theory of the large quark mass limit, it was observed that $\mathcal{C P}$-violating effects due a mixture of $\mathcal{C P}$-even and $\mathcal{C P}$-odd couplings leads to a phase shift of the $\phi_{j j}$ distribution compared to the $\mathcal{C P}$-even case by an angle, $\alpha$, which is given by the relative strength of the two couplings [24, 26]. Taking into account the relative enhancement by the factor 3/2 of the Agg coupling due to loop effects, the phase shift angle is given by

$$
\tan \alpha=\frac{3}{2} \frac{\tilde{y}_{q}}{y_{q}}
$$

when heavy quark loops of a single flavor dominate. In order to test this effect for the case of a light quark, we show, in the right panel of Fig. 8, the results for

$$
y_{b}=\frac{3}{2} \tilde{y}_{b}=-\tan \beta \frac{m_{b}}{v} \quad \text { and } \quad y_{t}=\frac{3}{2} \tilde{y}_{t}=-\cot \beta \frac{m_{t}}{v},
$$

where a 45 degree phase shift is expected, with minima of the $\phi_{j j}$ distribution at -45 and +135 degrees. This basic expectation is, indeed, confirmed by the detailed calculation. However, there are additional distortions of the azimuthal angle distributions which can again be explained by kinematical effects due to transverse momentum balancing of the two jets and the Higgs boson.

\section{Conclusions}

In this paper, we have presented the determination of quark mass effects on the cross section and on distributions for pseudoscalar Higgs production in association with two 

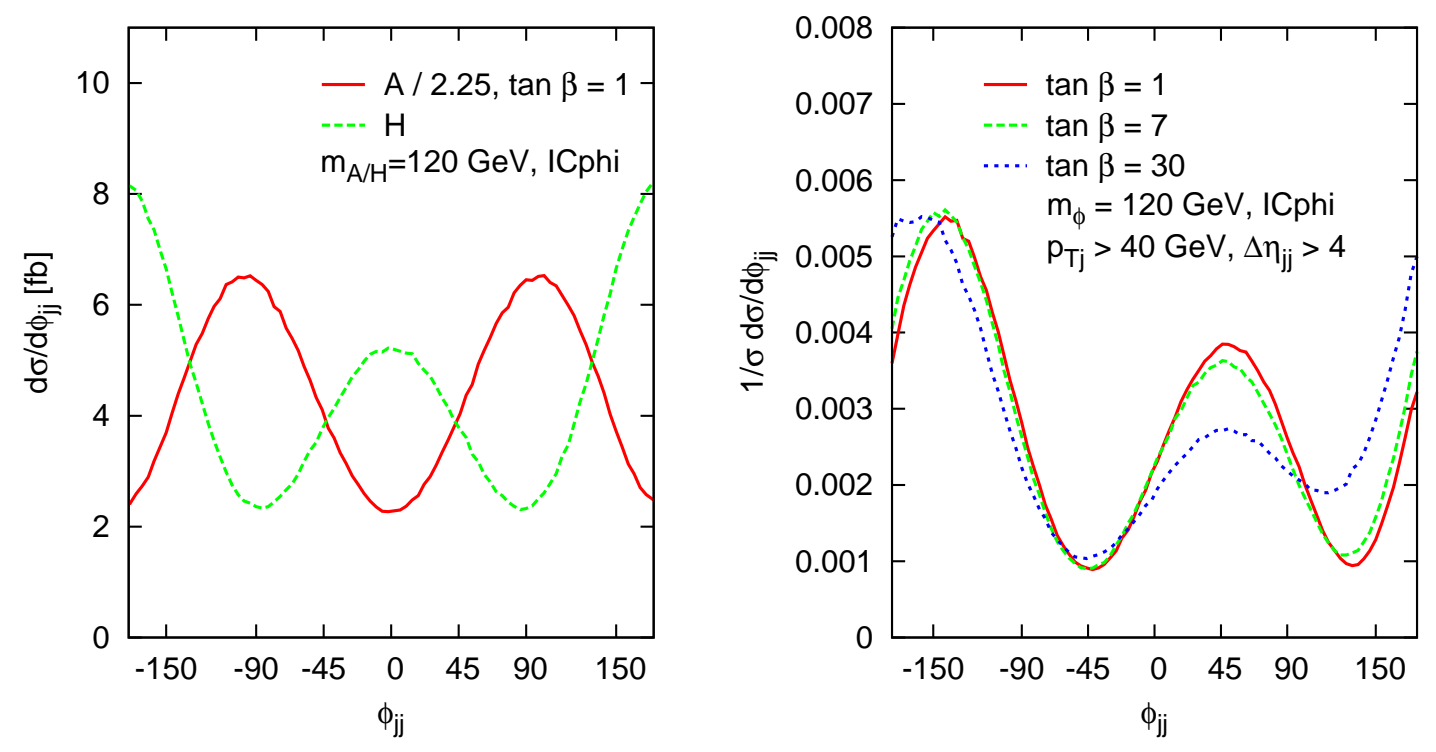

Figure 8: $\phi_{j j}$ distributions for different Higgs sector scenarios: pure $\mathcal{C} \mathcal{P}$-odd or $\mathcal{C P}$-even coupling in the effective Lagrangian limit (left panel) and the $\mathcal{C P}$ violating case defined in Eq. 4.9 (right panel). In the effective Lagrangian, the $\mathcal{C P}$-odd coupling was matched to the $\mathcal{C P}$-even coupling by a factor $2 / 3$. For the $\mathcal{C P}$-violating Higgs-sector, results are shown for different $\tan \beta$ values and with jet acceptance cuts tightened to $\Delta \eta_{j j}>4$ and $p_{T j}>40$ GeV as compared to the ICphi set (4.7).

final state partons. Our calculation for $A j j$ production complements the analogous one for a scalar Higgs, i.e. $H j j$ production as carried out in Ref. [1. Qualitative features are quite similar for the two cases. Validity of the heavy quark mass approximation is found to be restricted to $m_{\Phi}<m_{q}$ and $p_{T j}<m_{q}$ while large dijet invariant masses do not spoil the validity of the heavy quark limit. A pronounced difference between $A j j$ and $H j j$ production is observed in the azimuthal angle distribution between the two jets, which allows, in principle, to determine the $\mathcal{C P}$-properties of the produced Higgs boson at the LHC [26].

Our analytical expressions have been implemented in the VBFNLO program [10] and are publicly available as a parton level Monte Carlo program. Even though the code must evaluate loop expressions up to pentagons, the calculation is leading order in the strong coupling constant since Higgs production in gluon fusion first appears at the oneloop level. As a leading order process, it has been provided with an interface in the Les Houches format [28] to run with parton shower programs, providing full particle, momentum and color flow information. The code allows to sum top- and bottom-quark induced contributions with arbitrary $\mathcal{C P}$-violating couplings

$$
\mathcal{L}_{\text {Yukawa }}=\bar{q}\left(y_{q}+\mathrm{i} \gamma_{5} \tilde{y}_{q}\right) q \Phi
$$

and, thus, is versatile enough for simulating the effects of general, $\mathcal{C P}$-violating Higgs sectors at the LHC. 


\section{A Tensor structure of triangles}
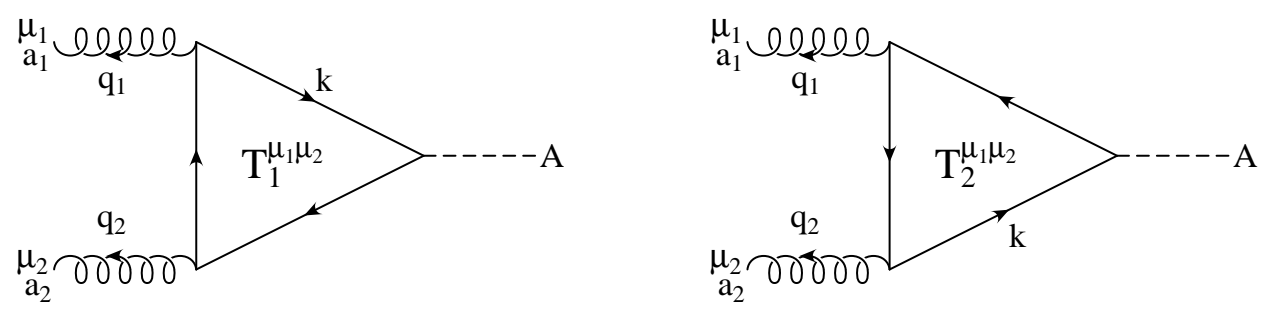

Figure 9: Two three-point functions connected by charge conjugation.

The generic three-point functions for triangle graphs with opposite loop momentum have the following expressions

$$
\begin{aligned}
& T_{1}^{\mu_{1} \mu_{2}}\left(q_{1}, q_{2}, m_{f}\right)=\frac{-\mathrm{i}}{4 m_{f}} \int \frac{\mathrm{d}^{4} k}{\mathrm{i} \pi^{2}} \operatorname{tr}\left[\frac{\not k+m_{f}}{k^{2}-m_{f}^{2}} \gamma^{\mu_{1}} \frac{\not k+\not 1+m_{f}}{\left(k+q_{1}\right)^{2}-m_{f}^{2}} \gamma^{\mu_{2}} \frac{\not k+q 12+m_{f}}{\left(k+q_{12}\right)^{2}-m_{f}^{2}} \gamma^{5}\right], \\
& T_{2}^{\mu_{1} \mu_{2}}\left(q_{1}, q_{2}, m_{f}\right)=\frac{-\mathrm{i}}{4 m_{f}} \int \frac{\mathrm{d}^{4} k}{\mathrm{i} \pi^{2}} \operatorname{tr}\left[\frac{\not k+m_{f}}{k^{2}-m_{f}^{2}} \gamma^{\mu_{2}} \frac{\not k+\not 2+m_{f}}{\left(k+q_{2}\right)^{2}-m_{f}^{2}} \gamma^{\mu_{1}} \frac{\not k+q 12+m_{f}}{\left(k+q_{12}\right)^{2}-m_{f}^{2}} \gamma^{5}\right]
\end{aligned}
$$

where $q_{1}, q_{2}$ are outgoing momenta, $q_{12}=q_{1}+q_{2}$ and the overall factor $-\mathrm{i} / 4 m_{f}$ cancels the explicit mass factor arising from the Dirac trace. Using the charge conjugation matrix C

$$
\hat{C} \gamma_{\mu} \hat{C}^{-1}=-\gamma_{\mu}^{T}, \quad \hat{C} \gamma_{5} \hat{C}^{-1}=\gamma_{5}^{T} \quad \text { with } \quad \hat{C}=\gamma^{0} \gamma^{2}, \quad \hat{C}^{2}=\mathbb{1}
$$

one can derive (Furry's theorem [12])

$$
T_{1}^{\mu_{1} \mu_{2}}\left(q_{1}, q_{2}, m_{f}\right)=T_{2}^{\mu_{1} \mu_{2}}\left(q_{1}, q_{2}, m_{f}\right) \equiv T^{\mu_{1} \mu_{2}}\left(q_{1}, q_{2}, m_{f}\right) .
$$

Thus, the color structure simplifies to

$$
\operatorname{tr}\left[t^{a_{1}} t^{a_{2}}\right] T_{1}^{\mu_{1} \mu_{2}}\left(q_{1}, q_{2}, m q\right)+\operatorname{tr}\left[t^{a_{2}} t^{a_{1}}\right] T_{2}^{\mu_{1} \mu_{2}}\left(q_{1}, q_{2}, m_{f}\right)=\delta^{a_{1} a_{2}} T^{\mu_{1} \mu_{2}}\left(q_{1}, q_{2}, m_{f}\right) .
$$

Evaluation of the Dirac trace yields

$$
T^{\mu_{1} \mu_{2}}\left(q_{1}, q_{2}, m_{f}\right)=\varepsilon^{\mu_{1} \mu_{2} q_{1} q_{2}} C_{0}\left(q_{1}, q_{2}, m_{f}\right) .
$$

Here, $C_{0}$ denotes the scalar three-point function and $\varepsilon^{\mu \nu q_{1} q_{2}}$ is the totally anti-symmetric tensor (Levi-Civita symbol), contracted with the gluon momenta $q_{1}$ and $q_{2}$. 

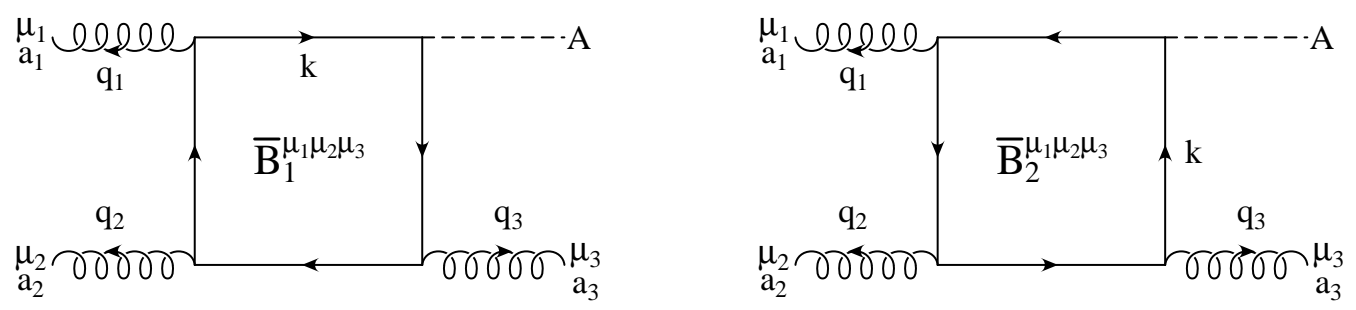

Figure 10: Two four-point functions connected by charge conjugation.

\section{B Tensor structure of boxes}

The analytic expressions for the charge-conjugated boxes are

$$
\begin{aligned}
\bar{B}_{1}^{\mu_{1} \mu_{2} \mu_{3}}\left(q_{1}, q_{2}, q_{3}, m_{f}\right)= & \frac{-\mathrm{i}}{4 m_{f}} \int \frac{\mathrm{d}^{4} k}{\mathrm{i} \pi^{2}} \operatorname{tr}\left[\frac{\not k+m_{f}}{k^{2}-m_{f}^{2}} \gamma^{\mu_{1}} \frac{\not k+\not q_{1}+m_{f}}{\left(k+q_{1}\right)^{2}-m_{f}^{2}}\right. \\
& \left.\times \gamma^{\mu_{2}} \frac{\not k+\not q_{12}+m_{f}}{\left(k+q_{12}\right)^{2}-m_{f}^{2}} \gamma^{\mu_{3}} \frac{\not k+\not q_{123}+m_{f}}{\left(k+q_{123}\right)^{2}-m_{f}^{2}} \gamma^{5}\right], \\
\bar{B}_{2}^{\mu_{1} \mu_{2} \mu_{3}}\left(q_{1}, q_{2}, q_{3}, m_{f}\right)= & \frac{-\mathrm{i}}{4 m_{f}} \int \frac{\mathrm{d}^{4} k}{\mathrm{i} \pi^{2}} \operatorname{tr}\left[\frac{\not k+m_{f}}{k^{2}-m_{f}^{2}} \gamma^{\mu_{3}} \frac{\not k+\not q_{3}+m_{f}}{\left(k+q_{3}\right)^{2}-m_{f}^{2}}\right. \\
& \left.\times \gamma^{\mu_{2}} \frac{\not k+\not q_{23}+m_{f}}{\left(k+q_{23}\right)^{2}-m_{f}^{2}} \gamma^{\mu_{1}} \frac{\not k+\not q_{123}+m_{f}}{\left(k+q_{123}\right)^{2}-m_{f}^{2}} \gamma^{5}\right],
\end{aligned}
$$

where $q_{1}, q_{2}$ and $q_{3}$ are outgoing momenta, $q_{i j}=q_{i}+q_{j}$ and $q_{i j k}=q_{i}+q_{j}+q_{k}$. From charge conjugation one gets

$$
\bar{B}_{1}^{\mu_{1} \mu_{2} \mu_{3}}\left(q_{1}, q_{2}, q_{3}, m_{f}\right)=-\bar{B}_{2}^{\mu_{1} \mu_{2} \mu_{3}}\left(q_{1}, q_{2}, q_{3}, m_{f}\right) \equiv \bar{B}^{\mu_{1} \mu_{2} \mu_{3}}\left(q_{1}, q_{2}, q_{3}, m_{f}\right) .
$$

Further two permutations are obtained by cyclic permutation of $(1,2,3)$. The color structure for the sum of the two diagrams is

$$
\begin{aligned}
& \operatorname{tr}\left(t^{a_{1}} t^{a_{2}} t^{a_{3}}\right) \bar{B}_{1}^{\mu_{1} \mu_{2} \mu_{3}}\left(q_{1}, q_{2}, q_{3}, m_{f}\right)+\operatorname{tr}\left(t^{a_{3}} t^{a_{2}} t^{a_{1}}\right) \bar{B}_{2}^{\mu_{1} \mu_{2} \mu_{3}}\left(q_{1}, q_{2}, q_{3}, m_{f}\right) \\
& \quad=\left[\operatorname{tr}\left(t^{a_{1}} t^{a_{2}} t^{a_{3}}\right)-\operatorname{tr}\left(t^{a_{3}} t^{a_{2}} t^{a_{1}}\right)\right] \bar{B}^{\mu_{1} \mu_{2} \mu_{3}}\left(q_{1}, q_{2}, q_{3}, m_{f}\right) \\
& \quad=\frac{\mathrm{i}}{2} f^{a_{1} a_{2} a_{3}} \bar{B}^{\mu_{1} \mu_{2} \mu_{3}}\left(q_{1}, q_{2}, q_{3}, m_{f}\right) .
\end{aligned}
$$


The tensor structure of charge-conjugation related box diagrams, e.g. with gluon permutation $(1,2,3)$, can be written as

$$
\begin{aligned}
& \bar{B}^{\mu_{1} \mu_{2} \mu_{3}}\left(q_{1}, q_{2}, q_{3}, m_{f}\right)=\left\{\varepsilon^{\mu_{3} q_{1} q_{2} q_{3}} g^{\mu_{1} \mu_{2}}-\varepsilon^{\mu_{2} q_{1} q_{2} q_{3}} g^{\mu_{1} \mu_{3}}+\varepsilon^{\mu_{2} \mu_{3} q_{2} q_{3}} q_{1}^{\mu_{1}}-\varepsilon^{\mu_{2} \mu_{3} q_{1} q_{3}} q_{2}^{\mu_{1}}\right. \\
& \quad+\varepsilon^{\mu_{2} \mu_{3} q_{1} q_{2}} q_{3}^{\mu_{1}}+\varepsilon^{\mu_{1} q_{1} q_{2} q_{3}} g^{\mu_{2} \mu_{3}}+\varepsilon^{\mu_{1} \mu_{3} q_{2} q_{3}} q_{1}^{\mu_{2}}-\varepsilon^{\mu_{1} \mu_{3} q_{1} q_{2}} q_{3}^{\mu_{2}}-\varepsilon^{\mu_{1} \mu_{2} q_{2} q_{3}} q_{1}^{\mu_{3}} \\
& \quad+\varepsilon^{\mu_{1} \mu_{2} q_{1} q_{3}} q_{2}^{\mu_{3}}+\varepsilon^{\mu_{1} \mu_{2} \mu_{3} q_{3}} g^{\mu_{1} \mu_{2}}-\varepsilon^{\mu_{1} \mu_{2} \mu_{3} q_{2}} g^{\mu_{1} \mu_{3}}+\varepsilon^{\mu_{1} \mu_{2} \mu_{3} q_{1}} g^{\mu_{2} \mu_{3}}+\varepsilon^{\mu_{1} \mu_{3} q_{1} q_{3}}\left(2 q_{1}^{\mu_{2}}\right. \\
& \left.\left.\quad+q_{2}^{\mu_{2}}\right)+\varepsilon^{\mu_{1} \mu_{2} q_{1} q_{2}}\left[2\left(q_{1}^{\mu_{3}}+q_{2}^{\mu_{3}}\right)+q_{3}^{\mu_{3}}\right]\right\} D_{0}\left(q_{1}, q_{2}, q_{3}, m_{f}\right) \\
& \quad-\varepsilon^{\mu_{1} \mu_{2} \mu_{3} q_{3}} C_{0}\left(q_{1}+q_{2}, q_{3}, m_{f}\right)-\varepsilon^{\mu_{1} \mu_{2} \mu_{3} q_{1}} C_{0}\left(q_{1}, q_{2}+q_{3}, m_{f}\right) \\
& +2 \varepsilon^{\mu_{2} \mu_{3} q_{2} q_{3}} D_{\mu_{1}}\left(q_{1}, q_{2}, q_{3}, m_{f}\right)+2 \varepsilon^{\mu_{1} \mu_{3} q_{1} q_{3}} D_{\mu_{2}}\left(q_{1}, q_{2}, q_{3}, m_{f}\right) \\
& \quad+2 \varepsilon^{\mu_{1} \mu_{2} q_{1} q_{2}} D_{\mu_{3}}\left(q_{1}, q_{2}, q_{3}, m_{f}\right) .
\end{aligned}
$$

The $D_{0}$ and $D_{\mu}$ are four-point functions. Whereas the former denotes a scalar function, the latter can be expressed by the usual Passarino-Veltman decomposition [14] as

$$
D^{\mu}\left(q_{1}, q_{2}, q_{3}, m_{f}\right)=q_{1}^{\mu} D_{11}+q_{2}^{\mu} D_{12}+q_{3}^{\mu} D_{13}
$$

Note that after contraction with polarization vectors $\epsilon_{1}^{\mu_{1}}, \epsilon_{2}^{\mu_{2}}$ and quark current $J_{21}^{\mu_{3}}$, the expression (B.5) still contains terms with factors $\left(\epsilon_{1} \cdot q_{1}\right),\left(\epsilon_{2} \cdot q_{2}\right),\left(J_{21} \cdot q_{3}\right)$ even though they vanish, since gluon polarization vectors $\epsilon_{i}^{\mu}$ and momenta $q_{i}^{\mu}$ are perpendicular to each other and the quark current $J_{21}$ is conserved. However, these terms are important for numerical gauge checks, where the corresponding gluon polarization vector is replaced by its momentum. Since the virtual gluon has a non-zero $q_{i}^{2}$, these terms give finite contributions.

\section{Tensor structure of pentagons}
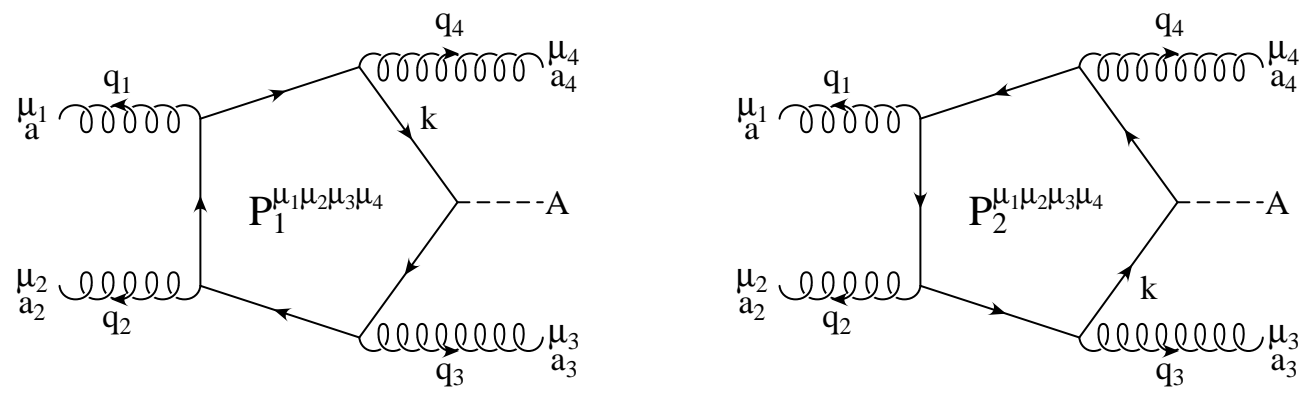

Figure 11: Two five-point functions connected by charge conjugation.

The two five-point functions connected by charge conjugation are defined by the ex- 
pressions

$$
\begin{aligned}
& P_{1}^{\mu_{1} \mu_{2} \mu_{3} \mu_{4}}\left(q_{1}, q_{2}, q_{3}, q_{4}, m_{f}\right)=\frac{-\mathrm{i}}{4 m_{f}} \int \frac{\mathrm{d}^{4} k}{\mathrm{i} \pi^{2}} \operatorname{tr}\left[\frac{\not k+m_{f}}{k^{2}-m_{f}^{2}} \gamma^{\mu_{4}} \frac{\not k+\not 4_{4}+m_{f}}{\left(k+q_{4}\right)^{2}-m_{f}^{2}} \gamma^{\mu_{1}}\right. \\
& \left.\times \frac{\not k+\not \not_{14}+m_{f}}{\left(k+q_{14}\right)^{2}-m_{f}^{2}} \gamma^{\mu_{2}} \frac{\not k+\not \not_{124}+m_{f}}{\left(k+q_{124}\right)^{2}-m_{f}^{2}} \gamma^{\mu_{3}} \frac{\not k+\not q_{1234}+m_{f}}{\left(k+q_{1234}\right)^{2}-m_{f}^{2}} \gamma^{5}\right] \text {, } \\
& P_{2}^{\mu_{1} \mu_{2} \mu_{3} \mu_{4}}\left(q_{1}, q_{2}, q_{3}, q_{4}, m_{f}\right)=\frac{-\mathrm{i}}{4 m_{f}} \int \frac{\mathrm{d}^{4} k}{\mathrm{i} \pi^{2}} \operatorname{tr}\left[\frac{\not k+m_{f}}{k^{2}-m_{f}^{2}} \gamma^{\mu_{3}} \frac{\not k+\not q_{3}+m_{f}}{\left(k+q_{3}\right)^{2}-m_{f}^{2}} \gamma^{\mu_{2}}\right. \\
& \left.\times \frac{\not k+\not_{23}+m_{f}}{\left(k+q_{23}\right)^{2}-m_{f}^{2}} \gamma^{\mu_{1}} \frac{\not k+\not \mathscr{q}_{123}+m_{f}}{\left(k+q_{123}\right)^{2}-m_{f}^{2}} \gamma^{\mu_{4}} \frac{\not k+\not_{1234}+m_{f}}{\left(k+q_{1234}\right)^{2}-m_{f}^{2}} \gamma^{5}\right] \text {, }
\end{aligned}
$$

where $q_{1}, q_{2}, q_{3}$ and $q_{4}$ are outgoing momenta $\left(q_{i j}=q_{i}+q_{j}\right.$ and similarly for $q_{i j k}$ and $\left.q_{i j k l}\right)$. The allowed color structures are given in [1]. The pentagon was reduced using the DennerDittmaier algorithm [18] and is available as a FORTRAN-subroutine in VBFNLO [17]. The full analytic expression in terms of Passarino-Veltman reduction can be found in [16].

\section{Acknowledgments}

We would like to thank Gunnar Klämke for helpful discussions, and Christian Schappacher, for the comparison with the FeynArts/FormCalc [15] framework. This research was supported in part by the Deutsche Forschungsgemeinschaft via the Sonderforschungsbereich/Transregio SFB/TR- 9 Computational Particle Physics. M.K. gratefully acknowledges support of the Graduiertenkolleg "High Energy Physics and Particle Astrophysics" and Landesgraduiertenförderung Baden-Württemberg. F.C acknowledges partial support by FEDER and Spanish MICINN under grant FPA2008-02878.

\section{References}

[1] V. Del Duca, W. Kilgore, C. Oleari, C. Schmidt and D. Zeppenfeld, Phys. Rev. Lett. 87 (2001) 122001 arXiv:hep-ph/0105129]; Nucl. Phys. B 616 (2001) 367 arXiv:hep-ph/0108030.

[2] G. L. Bayatian et al. [CMS Collaboration]. CMS physics: Technical design report.

[3] ATLAS detector and physics performance. Technical design report. Vol. 2, CERNLHCC-99-15, ATLAS-TDR-15

[4] A. Djouadi, Phys. Rept. 459 (2008) 1 arXiv:hep-ph/0503173].

[5] D. Zeppenfeld, R. Kinnunen, A. Nikitenko and E. Richter-Was, Phys. Rev. D 62 (2000) 013009 arXiv:hep-ph/0002036. 
[6] M. Duhrssen et al., Phys. Rev. D 70 (2004) 113009 [arXiv:hep-ph/0406323|.

[7] D. L. Rainwater and D. Zeppenfeld, JHEP 9712 (1997) 005 arXiv:hep-ph/9712271]; D. L. Rainwater, D. Zeppenfeld and K. Hagiwara, Phys. Rev. D 59 (1999) 014037 arXiv:hep-ph/9808468; D. L. Rainwater and D. Zeppenfeld, Phys. Rev. D 60 (1999) 113004 [Erratum-ibid. D 61 (2000) 099901] arXiv:hep-ph/9906218.

[8] R. P. Kauffman, S. V. Desai and D. Risal, Phys. Rev. D 55 (1997) 4005 [Erratum-ibid. D 58 (1998) 119901] arXiv:hep-ph/9610541.

[9] R. P. Kauffman and S. V. Desai, Phys. Rev. D 59 (1999) 057504 arXiv:hep-ph/9808286.

[10] K. Arnold et al., Comput. Phys. Commun. 180 (2009) 1661 arXiv:0811.4559, hep$\mathrm{ph}$.

[11] J. F. Gunion, H. E. Haber, G. L. Kane and S. Dawson, The Higgs Hunter's Guide, Addison-Wesley, Reading (USA), 1990.

[12] W. H. Furry, Phys. Rev. 51 (1936) 125.

[13] K. Hagiwara and D. Zeppenfeld, Nucl. Phys. B 313 (1989) 560.

[14] G. Passarino and M. J. G. Veltman, Nucl. Phys. B 160 (1979) 151.

[15] T. Hahn, Comput. Phys. Commun. 140 (2001) 418 arXiv:hep-ph/0012260; T. Hahn and C. Schappacher, Comput. Phys. Commun. 143 (2002) 54 [hep-ph/0105349]; T. Hahn, M. Perez-Victoria, Comput. Phys. Commun. 118 (1999) 153 [hep-ph/9807565

[16] M. Kubocz, Diploma thesis (german), Institut für Theoretische Physik, Universität Karlsruhe, 2006, http://www-itp.particle.uni-karlsruhe.de/diplomatheses.de.shtml

[17] The VBFNLO code can be obtained from http://www-itp.particle.uni-karlsruhe.de/vbfnlo/

[18] A. Denner and S. Dittmaier, Nucl. Phys. B 658 (2003) 175 arXiv:hep-ph/0212259; Nucl. Phys. B 734 (2006) 62 arXiv:hep-ph/0509141.

[19] J. Pumplin, D. R. Stump, J. Huston, H. L. Lai, P. Nadolsky and W. K. Tung, JHEP 0207 (2002) 012 arXiv:hep-ph/0201195|.

[20] M. Spira, Fortsch. Phys. 46 (1998) 203 arXiv:hep-ph/9705337.

[21] J. A. M. Vermaseren, S. A. Larin and T. van Ritbergen, Phys. Lett. B 405 (1997) 327 arXiv:hep-ph/9703284.

[22] T. Plehn, D. L. Rainwater and D. Zeppenfeld, Phys. Rev. Lett. 88 (2002) 051801 arXiv:hep-ph/0105325. 
[23] V. Hankele, G. Klamke and D. Zeppenfeld, "Higgs +2 jets as a probe for CP properties," arXiv:hep-ph/0605117].

[24] V. Hankele, G. Klamke, D. Zeppenfeld and T. Figy, Phys. Rev. D 74 (2006) 095001 arXiv:hep-ph/0609075.

[25] G. Klamke and D. Zeppenfeld, "Hjj production: Signals and CP measurements," arXiv:0705.2983, hep-ph].

[26] G. Klamke and D. Zeppenfeld, JHEP 0704 (2007) 052 arXiv:hep-ph/0703202.

[27] J. M. Campbell, R. K. Ellis and G. Zanderighi, JHEP 0610 (2006) 028 arXiv:hep-ph/0608194.

[28] E. Boos et al., arXiv:hep-ph/0109068; J. Alwall et al., Comput. Phys. Commun. 176 (2007) 300 arXiv:hep-ph/0609017. 